\section{OSCILLATORY MOMENTUM TRANSPORT IN CASCADE TRANSITIONAL BOUNDARY LAYER FLOWS}

\author{
Luis R. Rojas-Solórzano* \\ Universidad Simón Bolívar \\ Caracas. Venezuela. A.P. 89000
}

\section{ABSTRACT}

The generation and early evolution of boundary layer transitional instabilities, named TollmienSchlichting (T-S) waves, in airfoil cascade flows are studied. The energy exchange between the mean flow and the flow instabilities is computed by performing Direct Numerical Simulation of the fluid flow governing equations and by calculating the fluctuating kinetic energy (FKE) budget within the separated boundary layer. The driving role of the FKE production in the wavelength modulation process associated to the receptivity phenomenon, i.e., the generation of T-S waves, is demonstrated. The FKE production largely hastens the wavelength modulation around the inflection point of the mean velocity profile across the boundary layer. Above the inflection point, the fluctuating pressure field favors the energy transport and provides the energy necessary to convect the instabilities out of the boundary layer. The evolution of the T-S waves depicts an asymmetric distribution of the production term in the transverse direction, i.e., in the lower half of the boundary layer the mean flow provides energy to the instabilities while the opposite occurs in the upper half.

\section{INTRODUCTION}

In a recent numerical study, Rojas \& Amon (1997) explored the subcritical and supercritical boundary layer receptivity and stability in unperturbed and perturbed flows within airfoil cascades for low Reynolds numbers (Re). Receptivity is understood as the physical mechanism by which energy is transferred from the typically long wavelength free-stream disturbances to the short wavelength boundary layer transitional instabilities (Morkovin, 1969). The stability of the boundary layer depends on the attenuation or promotion of the primary transitional waves, known as Tollmien-Schlichting (T-S) waves. Rojas \& Amon (1997) found that for the unperturbed flows, above a critical Reynolds number $R_{c}$, the boundary layer separates and becomes unsteady.

\footnotetext{
* Profesor Agregado. Dpto. de Conversión y Transporte de Energía. "Copyright (C) 1998 by the American Institute of Aeronautics and Astronautics, Inc. All rights reserved."
}

AIAA-98-2560

Through the visualization of the instability evolution, it was concluded that the boundary layer separation provided the rapid and localized change in the mean flow capable to trigger the generation of primary transitional instabilities, or receptivity. That work (Rojas \& Amon, 1997), provided an explanation to the receptivity phenomenon and to the evolution of the T-S waves based on direct observation of the instantaneous flow field and the fluctuating component of the flow. The observations revealed the refractory nature of the boundary layer receptivity as the bubble within the separated boundary layer offered an increased resistance to the instability convection and forced the wave speed reduction while keeping the wave frequency constant. The wave speed reduction explains the consequent wavelength modulation necessary for the generation of the T-S waves.

The main objective of this work is to study the boundary layer receptivity and stability through a nonclassical approach. This investigation is performed through the evaluation and analysis of the fluctuating kinetic energy (FKE) budget of the velocity and pressure fluctuations computed using Direct Numerical Simulations (DNS). DNS is a valuable tool to study incompressible flows since no assumptions are necessary to solve the full Navier-Stokes and mass conservation equations. The DNS here proposed is based on spectral element spatial discretization which provided the necessary high accuracy to solve the steep gradients of the pressure and velocity fields that develop within the boundary layer. The FKE budget, though traditionally used in the evaluation of turbulent flows, has proven to be useful in the investigation of different energy transfer mechanisms between the mean and the fluctuating flow in weakly transitional regimes (Majumdar \& Amon, 1997). Majumdar \& Amon (1997) recently presented results of the study of the oscillatory momentum transport in transitional flows within communicating channels. Their objective was to identify mechanisms responsible for sustaining the fluctuating flow by evaluating the FKE terms. They found that the pressure fluctuation and the production terms are mainly responsible for the exchange of energy between the mean and the fluctuating flow. In particular, the pressure fluctuation contributed to sustaining the flow fluctuations in the vortical communicating region, whereas the production term was mainly responsible for sustaining the FKE in the near-parallel channel flow.

\section{FKE Budget}

The kinetic energy equation for the fluctuating components of the velocity and pressure (1) results 
from the time-averaged manipulation of the momentum and mass conservation equations, starting from the Reynolds decomposition (Hinze, 1987). The equation (1) represents the conservation of FKE in the elementary control volume depicted in Fig. 1 (Bradshaw, 1975). The equation (1) states that within an elementary control volume, the balance among the diffusive transport of FKE by the fluctuations, the work of the fluctuations to diffuse through the viscous field, the production and dissipation must equal the time rate of increase of FKE and the convection of FKE by the mean flow. This equation is expressed in nondimensional form as:

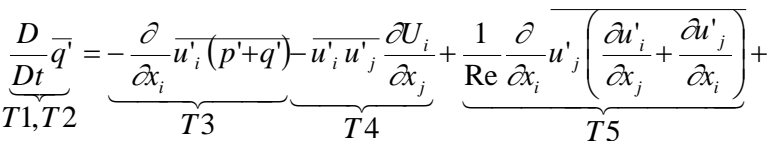

$$
\begin{aligned}
& \Downarrow \\
& \underbrace{-\frac{1}{\operatorname{Re}}\left(\frac{\partial u_{i}^{\prime}}{\partial x_{j}}+\frac{\partial u_{j}^{\prime}}{\partial x_{i}}\right) \frac{\partial u_{j}^{\prime}}{\partial x_{i}}}_{T 6}
\end{aligned}
$$

$$
\begin{aligned}
T 1 & =\frac{\partial}{\partial t}\left(\frac{u_{i}^{\prime} u_{i}^{\prime}}{2}\right) \\
\mathrm{T} 2 & =\frac{\partial}{\partial x_{\mathrm{i}}}\left(U_{i} \frac{u_{j}^{\prime} u_{j}^{\prime}}{2}\right)
\end{aligned}
$$

where,

q' : fluctuating kinetic energy $\mathrm{FKE}=\left(\mathrm{u}_{1},{ }^{2}+\mathrm{u}_{2}{ }^{, 2}\right) / 2$

$-\mathrm{u}_{\mathrm{i}}^{\prime} \mathrm{u}_{\mathrm{j}}^{\prime}$ : fluctuating (Reynolds) stress

$\mathrm{U}_{\mathrm{i}}$ : time-averaged velocity

T1 : time rate of increase of FKE

T2 : convective diffusion of FKE by the mean flow

T3 : work of the total dynamic pressure of the fluctuations

T4 : production of FKE

T5 : work of the viscous shear stresses of the fluctuations

T6 : viscous dissipation of FKE

Terms T1-T6 are non-dimensionalized by $c / U_{\infty}^{3}$.

We validated the kinetic energy subroutine in two ways. First, the sum of the fluctuating components of the velocity, $\Sigma u^{\prime}$ and $\Sigma v$ ', within one period of the flow fluctuation must be very small (e.g., in the order of the machine round-off error); and second, the sum of terms T3 through T6 must be approximately, within the round-off error of the machine, equal to T2. The second validation was performed within the suctionside boundary layer and the quasi-potential region between the two airfoils. The validation confirmed that $\mathrm{T} 1$ is neglegible as it should be for asymptotically converged flows.

From the expressions of the energy terms in eqn. (1), it is possible to predict some features of the energy exchange within the flow. For example, the production of FKE, T4, may be affected in two different ways : when $i \neq j, T 4$ usually gives a positive contribution to the kinetic energy of the instabilities (Hinze, 1987). Lin (1955) demonstrated that viscosity effects at the wall lead to a phase shift between the streamwise and the transversal velocity components of a twodimensional disturbance, and consequently to a positive value of the Reynolds shear stress $-\rho \overline{u_{1} u_{2}^{\prime}}$. Since close to the wall, except for separated regions, $\partial \mathrm{U}_{1} / \partial \mathrm{x}_{2}>0$, a positive value of the production $\mathrm{T} 4$ is expected near the airfoil surfaces within the boundary layer.

When $i=j, T 4$ tends to be negative for spatially accelerated flows and positive for retarded flows; therefore, a decrease of static pressure in the flow direction (favorable pressure gradient) inhibits the fluctuations and an increase in static pressure (adverse pressure gradient) promotes fluctuations.

\section{Mathematical Model}

MODELING AND APPROACH

The geometry under consideration, depicted in Fig. 2, corresponds to the midspan airfoil-to-airfoil surface of an experimental stator row (Dring, Blair, Joslyn, Power \& Verdon, 1988). The governing equations are the incompressible two-dimensional Navier-Stokes and conservation of mass equations:

$$
\begin{aligned}
& \frac{\partial \vec{u}}{\partial t}+\vec{u} . \nabla \vec{u}=-\nabla p+\frac{1}{\operatorname{Re}} \nabla^{2} \vec{u} \quad \text { in } \partial \mathrm{D} \\
& \nabla \cdot \vec{u}=0 \text { in } \partial \mathrm{D}
\end{aligned}
$$

where, $\mathrm{x}=\mathrm{x} * / \mathrm{c}, \mathrm{y}=\mathrm{y} * / \mathrm{c}, \mathrm{u}=\mathrm{u}^{*} / \mathrm{U}_{\infty}, \mathrm{v}=\mathrm{v}^{*} / \mathrm{U}_{\infty}, \mathrm{p}=$ $\mathrm{p} * / \rho . \mathrm{U}_{\infty}{ }^{2}, \mathrm{t}=\mathrm{t}^{*} . \mathrm{U}_{\infty} / \mathrm{c}$ and subject to the following boundary conditions:

a) inflow boundary conditions

$$
u_{H-A}=1 ; \mathrm{v}_{H-A}=0
$$

b) outflow boundary conditions

$$
\begin{aligned}
& \frac{\partial u}{\partial x}=0 \\
& \frac{\partial v}{\partial x}+\frac{\partial u}{\partial y_{(D-E)}}=0
\end{aligned}
$$

c) blade wall

$$
u_{B-C}=u_{G-F}=0
$$

d) meridional bounds

$$
\begin{aligned}
& u_{A-B}=u_{G-H} ; u_{C-D}=u_{E-F} \\
& \mathrm{v}_{A-B}=\mathrm{v}_{G-H} ; \mathrm{v}_{C-D}=\mathrm{v}_{E-F}
\end{aligned}
$$

$(\partial \mathrm{D}$ is the computational domain with the reference corners A through $\mathrm{H}$ shown in Fig. 2) 
Further details of the temporal and spatial discretization for this geometry can be found in Rojas \& Amon (1995).

\section{Numerical Approach}

DNS is used to solve the discretized governing equations. The spatial discretization of the domain is conducted using the spectral element technique (Patera, 1984; Korczag \& Patera, 1986; Amon, 1993). The spectral element technique is a high-order, weighted-residual technique that combines the geometric flexibility of the finite element technique with the accuracy and rapid convergence of spectral methods.

The temporal discretization of the Navier-Stokes equations must accomplish three major objectives. First, limitations in computer time suggest that the non-linearities be treated explicitly, i.e, the convective term should be calculated from values of the velocity from the previous time-steps. Second, the viscous term should be treated implicitly to avoid unreasonable time-step restrictions. These time-steps restrictions would be prohibitive because of the high resolution of spectral techniques adjacent to boundaries (Gottlieb \& Orszag, 1977). Finally, the pressure should also be calculated implicitly, because of the divergence-free velocity imposed at each time-step. In response to these needs, a time-stepping scheme based upon a consistent choice of approximation spaces for the pressure and velocity within the semi-discrete formulation of the time-dependent term in the Navier-Stokes equations is used. The complete solution of the NavierStokes equations involves first treating the wave-like equation for the non-linear convective terms explicitly, and then solving the resultant Stokes problem at each time-step by the Uzawa iterative procedure (Rønquist, 1988).

Details of the formulation and the methodology may be encountered in Rojas \& Amon (1995).

The spectral mesh with 784 elements and 25 collocation points per element is depicted in Fig. 3 for three contiguous blades.

\section{Calculation of the FKE Terms}

The FKE terms are determined through the spectral evaluation of the velocity and pressure spatial derivatives. Lagrangian-Legendre's interpolants are used in the expansions for the velocity and pressure. For instance, any of the scalar components of the problem ( $\mathrm{x}$-velocity, $\mathrm{y}$-velocity and pressure) can be represented as a $\mathrm{N}^{\text {th }}$-order polynomial, $\mathrm{g}(\xi)$ on a local domain, simplified for practical purposes to onedimension as $\xi \in[-1,1]$. This polynomial is expanded within the code in the finite series:

$$
g(\xi)=\sum_{j=0}^{N} h_{j}(\xi) g\left(\xi_{j}\right)
$$

The Lagrangian interpolants, $\mathrm{h}_{\mathrm{j}}(\xi)$ are $\mathrm{N}^{\mathrm{th}}$-order polynomials such that :

$$
h_{j}\left(\xi_{i}\right)=\delta_{i j} \text { for all } \mathrm{i}, \mathrm{j} \in\{0, \ldots, \mathrm{N}\}^{2}
$$

That is, each interpolant is 1 at one collocation point and 0 at all other collocation points.

The nodal Lagrangian-Legendre interpolants, $h_{j}$, can be written as :

$$
h_{j}(\xi)=\frac{\left(1-\xi^{2}\right) L_{N}^{\prime}(\xi)}{N(N+1) L_{N}\left(\xi_{j}\right)\left(\xi-\xi_{j}\right)}
$$

where $L_{k}(\xi), \quad k=1,2,3, \ldots \quad$ is the Legendre polynomial and prime denotes differentiation.

The Gauss-Lobatto-Legendre collocation points are related to the actual coordinates $\mathrm{x}$ and $\mathrm{y}$ by :

$\xi_{0}=-1$

$\xi_{i}(i=1, \ldots, N-1)=$ zeroes of $L_{N}^{\prime}$

$\xi_{N}=1$

With this expression relating the local and global coordinates, the spatial derivatives are computed as a function of the local derivatives. The first local derivative of the polynomial $\mathrm{g}(\xi)$ at the nodal points $\xi_{\mathrm{i}}$ is given by:

$$
g^{\prime}\left(\xi_{i}\right)=\sum_{j=0}^{N} h_{j}\left(\xi_{i}\right) g\left(\xi_{j}\right)=\sum_{j=0}^{N} D_{i j} g_{j}
$$

where $g_{j}=g\left(\xi_{j}\right)$, prime denotes differentiation and the nodal interpolant derivative matrix, $\mathrm{D}$, is defined as:

$$
D_{i j}=\frac{d h_{j}}{d \xi}\left(\xi_{i}\right)
$$

\section{NUMERICAL RESULTS}

The next sub-sections describe the numerical results obtained for a self-sustained fluctuating basic flow at $\operatorname{Re}=1000$ ( $\operatorname{Re}$ based on the blade chord and free-stream velocity.) The objective of the analysis is to explain the boundary layer receptivity and stability characteristics within self-sustained oscillatory basic flows. We include results based on the mean flow vorticity, viscous shear stress, Reynolds stress and the FKE itself to help in the interpretation of the FKE budget. Figure 4 depicts the airfoil with locations along the suction side and normal direction to the wall at 
different positions downstream the leading edge, as they will be referred to in the figures.

\section{Mean Flow Characteristics}

Figures 5a through $5 \mathrm{~h}$ show the plots of the tangential velocity, vorticity, shear stress, Reynolds shear stress and fluctuating kinetic energy at different perpendicular locations along the suction-side boundary layer under favorable pressure gradient (FPG) and adverse pressure gradient (APG). The regions under FPG and APG are separated in the figures by the minimum pressure point (MPP). The section of the boundary layer under FPG is characterized by the largest vorticity and shear stress on the wall, although these two parameters diminish steeply when approaching the boundary layer outer region. The section of the airfoil under APG is characterized, as expected, for presenting an inflection point in the velocity profile at every transversal station. Since the flow close to the airfoil wall has a large component parallel to it, the vorticity $\left(\omega_{z}\right)$ and shear stress $\left(\tau_{\mathrm{xy}}\right)$ are generated at the wall and maximized around the inflection point. The time-averaged kinetic energy of the fluctuations $\bar{q}$ rapidly increases as the flow convects from the leading edge towards the trailing edge. Under APG, as expected, $\bar{q}$ is enhanced faster and preferentially towards the boundary layer outer edge as a consequence of the interaction with the wake instabilities. The intensity of the Reynolds stresses $\overline{u_{t}^{\prime} u_{n}^{\prime}}$ is similarly enhanced towards the trailing edge, though under APG its distribution alternates from positive and moderate close to the wall, as predicted by Lin (1955), to negative and large towards the outer edge of the boundary layer.

\section{Time-average Equilibrium of the Flow}

The calculation of the FKE terms T2-T6 is accomplished within the entire domain. However some scatter in the pressure term (T3) product of the large gradients and the discontinuity in the derivatives across the boundary of contiguous elements suggested to calculate the pressure term as the balance of the FKE. However, for this calculation to be accurate, it requires that T1 be negligible. Figure 6 shows (a) T3 directly computed from the fluctuating velocity and pressure fields; and (b) T3 obtained from the balance of the FKE equation, neglecting $\mathrm{T} 1$. In the comparison, T3 is shown within the airfoil suction-side boundary layer. The comparison shows that T3 obtained from the balance of the FKE equation, while smoothing out the scatter in the computed $\mathrm{T} 3$, reproduces accurately the main features of the computed term. Neglecting T1 is consistent with the fact that although we are considering unsteady flows, in time-average, the flow is steady and in equilibrium without localized gain or loss of FKE. From now on, the T3 shown in the FKE budgets is the calculated to balance the equation, while $\mathrm{T} 1$ is assumed to be zero everywhere.

\section{FKE Budgets}

Figure 7 shows the FKE budget for $\mathrm{Re}=1000$ in five different stations: beyond the leading edge $\left(\mathrm{L}_{3}\right)$, downstream the minimum pressure point -MPP- $\left(\mathrm{L}_{5}\right)$, at the separation point $\left(\mathrm{L}_{6}\right)$, in the middle of the separated bubble $\left(\mathrm{L}_{7}\right)$ and close to the trailing edge $\left(\mathrm{L}_{8}\right)$. The FKE budgets are presented as a function of the normal distance to the wall $d_{n}$, nondimensionalized by the local boundary layer thickness $\delta$. At the entrance $\left(\mathrm{L}_{3}\right)$, moderate dissipation of FKE (T6) over the wall is accompanied by a favorable fluctuating pressure term (T3) which provides FKE uniformly across the section to sustain the convection (T2) of the long wavelength instability generated at the leading edge. The production term (T4), as expected under FPG, is almost negligible within the lower half of the boundary layer, while negative within the upper half. A negative T4 indicates that the mean flow withdraws energy from the instability and promotes its attenuation. Between the MPP and the separation point (SP) at $\mathrm{L}_{5}$, the FKE budget describes the energy transport during the wavelength modulation process that gives rise to the receptivity phenomenon, i.e., the generation of $\mathrm{T}-\mathrm{S}$ waves. While the fluctuating pressure field (T3) offers resistance to the convection of the instability within the lower half of the boundary layer, the production is intensive around the inflection point and assists the wavelength modulation. The largest production of FKE around the inflection point reflects the highest level of shear stress and vorticity we observed around the inflection point in the mean flow (see Fig. 5e). Since the shear stress and the vorticity are measures of the level of deformation and rotational velocity of fluid elements, respectively, it is expected that the largest deformation of the mean flow will cause the largest amount of work against the fluctuating shear stresses, i.e., T4. Within the boundary layer upper half, the production becomes negligible and the fluctuating pressure (T3) becomes the driving force that sustains the instability convection along with the mean flow (T2). The distribution of the Reynolds stresses across the station, shown in Fig. 8, confirms that the largest momentum exchange of the fluid fluctuation in the tangential and normal direction $\overline{u_{t} u_{n}^{\prime}}$ occurs towards the outer edge of the boundary layer.

To further the study of the receptivity using the FKE terms, we plot the distribution of T2, T3 and T4 over the area where the wavelength modulation occurs. Figures 9a, 9b and 9c depict the distribution of T2, T3 
and $\mathrm{T} 4$, respectively, within the airfoil suction-side boundary layer between the MPP and the SP. The three figures show characteristic behaviors around the inflection point of the velocity profile, which is located at about $0.3 \delta$ from the wall in the middle of the section between the MPP and the SP. The wavelength modulation is marked by a progressive reduction of the instability convection (T2) reaching a minimum in the middle of the section and followed by a progressive recovery towards the SP. The fluctuating pressure field (T3), while contributing moderately to the instability convection within a sub-layer very close to the wall, offers resistance to the instability development around the inflection point and probably is the opposite force that explains the reduction of wave phase speed that forces the refraction of the wave. The production term T4, while intense around the MPP and immediately downstream the SP, is reduced considerably towards the middle of the section between the MPP and the SP. The reduction of the production of FKE during the intermediate phase of the wavelength modulation might result from the instability wavelength contraction that is done against the mean flow and therefore, while in process, requires the transport of FKE to the mean flow.

The characteristics of the T-S wave evolution are understood from the observations at stations $\mathrm{L}_{6}, \mathrm{~L}_{7}$ and $\mathrm{L}_{8}$. The FKE budgets at stations $\mathrm{L}_{6}, \mathrm{~L}_{7}$ and $\mathrm{L}_{8}$, show the FKE transport at the SP, the middle of the bubble and further downstream close to the trailing edge, respectively, as shown in Fig. 7. The FKE budgets at these three stations are qualitatively similar and quantitatively larger for the stations $\mathrm{L}_{7}$ and $\mathrm{L}_{8}$ compared to the station $\mathrm{L}_{6}$. The larger energy transport at $\mathrm{L}_{7}$ and $\mathrm{L}_{8}$ obeys to the evolution of T-S waves within the bubble. At all three locations, both $\omega_{z}$ and $\tau_{x y}$ are maxima around the IP as seen in Figs. 5f through $5 \mathrm{~h}$. Within the lower half of the boundary layer the vorticity increases towards the center, and the unsteady pressure gradient (T3) offers moderate resistance to the FKE transport. The FKE production (T4) within the separated boundary layer shows a diffusive distribution indicating that energy is transferred within the lower half to account for the convection along the mean flow, while energy is released back to the mean flow by about the same proportion within the upper half. Within the upper half of the boundary layer the convection of the instability along with the mean flow (T2) is proportional to the distance from the wall and is promoted by the fluctuating pressure gradient (T3) in the transversal direction. Therefore the propagation of T-S waves within the airfoil boundary layer can be seen as promoted by two agents: within the boundary layer lower half, the propagation of $\mathrm{T}-\mathrm{S}$ waves is supported by the work of deformation-restoration of the mean flow under adverse pressure gradient against the fluctuating stresses (T4), while within the upper half, the T-S wave propagation is sustained by the pressure fluctuations which assist the wave convection and the transfer of energy to the mean flow.

\section{CONCLUDING REMARKS}

The mechanisms of energy exchange and transformation between the mean flow and the fluctuations in the Fluctuating Kinetic Energy (FKE) equation are computed for a weakly transitional boundary layer flow within an experimental airfoil cascade. Particular attention is given to the analysis of the FKE budgets within the airfoil suction-side boundary layer to explain the receptivity phenomenon and the stability characteristics.

For unsteady basic flows at $\mathrm{Re}=1000$, the initiation of the instability wavelength modulation process, marked by the minimum pressure point, is accompanied by the production of FKE predominantly around the mean velocity inflection point. Above and underneath the inflection point, the production decays gradually towards the boundary layer edges. The production of FKE mainly accounts for the energy demanded to convect the instability against an adverse fluctuating pressure gradient while the wavelength contraction is taking place. Above the inflection point, the fluctuating pressure gradient favors the convection of instabilities out of the boundary layer. The evolution of T-S waves within the separated bubble is characterized by a transverse asymmetric production of FKE; positive within the lower half of the boundary layer and negative within the upper half. The positive production reaches its maximum around the inflection point. Within the upper half of the boundary layer, the $\mathrm{T}-\mathrm{S}$ wave convection is intensified by the action of the fluctuating pressure field.

\section{REFERENCES}

Amon, C.H., 1993, "Spectral Element-Fourier Method for Transitional Flows in Complex Geometries," AIAA Journal, Vol. 31, No. 1, pp. 42-48.

Amon, C.H. and Patera, A.T., 1989, "Numerical Calculation of Stable Three-Dimensional Tertiary States in Grooved-Channel Flow," Physics of Fluids A, Vol. 1 (12), pp. 2005-2011.

Drazin, P.G. and Reid, W.H., 1991, "Hydrodynamic Stability," Cambridge University Press, USA.

Dring, R.P., Blair, M.F., Joslyn, H.D., Power, G.D. and Verdon, J.M., 1988, "The Effects of Inlet Turbulence and Rotor/Stator Interactions on the Aerodynamics and Heat Transfer of a Large-Scale 
Rotating Turbine Model, I-Final Report," NASA Contractor Report 4079.

Dring, R.P., Joslyn, H.D., Hardin, L.W. and Wagner, J.H., 1982, "Turbine Rotor-Stator Interaction," Journal of Engineering for Power, Vol. 104, pp. 729-742.

Fischer, P., 1989, "Spectral Element Solution of the Navier-Stokes Equations on High Performance Distributed-Memory Parallel Processors," PhD. Thesis, Massachusetts Institute of Technology.

Gaster, M., 1969, "The Structure and Behavior of Laminar Separation Bubbles," ARC R\&M 3595.

Gottlieb, D. and Orszag, S.A., 1977, "Numerical Analysis of Spectral Methods. Theory and Applications," Vol. 26, CBMS-NSF Monograph SIAM, Philadelphia.

Hinze, J.O., 1987, "Turbulence," McGraw-Hill Publishing Company, 2nd ed., New York.

Lin, C.C., 1955, "The Theory of Hydrodynamic Stability," Cambridge University Press, New York.

Majumdar, D., 1993, "Fluid Flow and Heat Transfer Mechanisms of Self-Sustained Oscillations in Communicating Channels," Ph.D. Thesis, Carnegie Mellon University.

Majumdar, D. and Amon, C.H., 1997, "Oscillatory Momentum Transport Mechanisms in Transitional Complex Geometry Flows," Journal of Fluids Engineering, Vol. 119, pp. 1-7.

Morkovin, M.V., 1969, "Critical Evaluation of Transition from Laminar to Turbulent Shear Layer with Emphasis on Hypersonically Traveling Bodies," Air Force Flight Dyn. Lab. Rep. AFFDL-TR-68-149 (Available as NTIS AD-686178).

Patera, A.T., 1984, "A Spectral Element Method for Fluid Dynamics: Laminar Flow in a Channel Expansion," Journal of Computational Physics, Vol. 54, No. 3, pp. 468-488.

Renaud, E.W., 1991, "Secondary Flow, Total Pressure Loss and the Effect of Circumferential Distortions in Axial Turbine Cascades," Ph.D. Thesis, Massachusetts Institute of Technology.

Rojas, L.R. and Amon, C.H., 1995, "Direct Numerical Simulations of Boundary Layer Instabilities in Subsonic Cascade Flows Triggered by Free-Stream Disturbances," Proceedings of the Third Caribbean Congress on Fluid Dynamics and the Third LatinAmerican Symposium on Fluid Mechanics, Caracas, Venezuela.

Rojas, L.R. and Amon, C.H., 1997, "Resonant Interaction Between Boundary Layer Instabilities and Free-Stream Disturbances in Cascade Flows," to be submitted to Journal of Fluids Engineering.

Rønquist, E., 1988, "Optimal Spectral Element Methods for the Unsteady Three-Dimensional
Incompressible Navier-Stokes Equations," PhD. Thesis, Massachusetts Institute of Technology.

Schlichting, H., 1979, "Boundary-Layer Theory," McGraw-Hill Publishing Company, 7th ed., New York.

Temam, R., 1977, "Navier-Stokes Equations," NorthHolland, Amsterdam.

Wlezien, R. W., "Meas rement of Boundary Layer Receptivity: Challenges and Frontiers," ASME: FED Transitional and Turbulent Compressible Flows, Vol. 224, pp. 1-8, 1995.

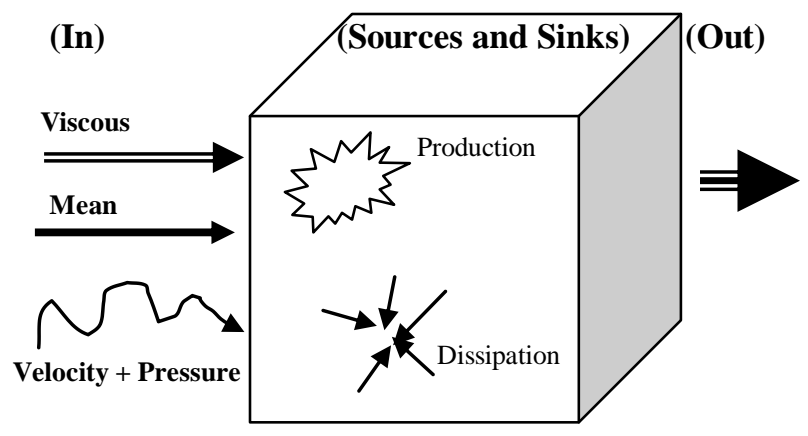

Fig 1 Balance of FKE in elementary control volume

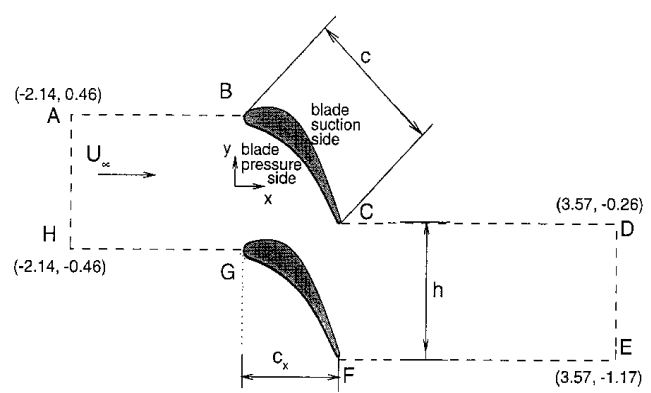

Fig. 2 Two-dimensional computational domain 


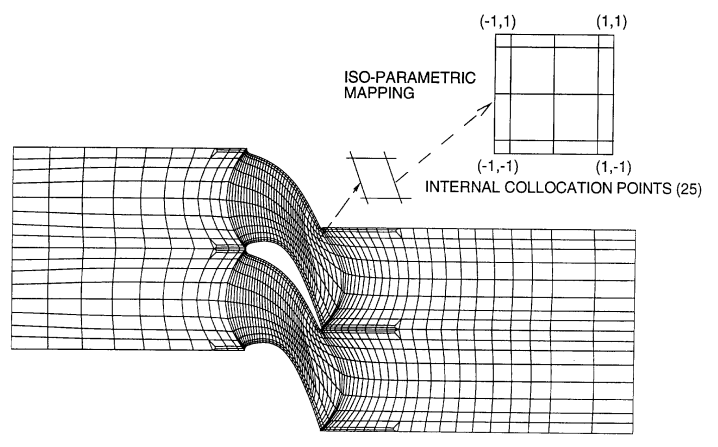

Fig. 3 Mesh discretization. 784 macro-elements

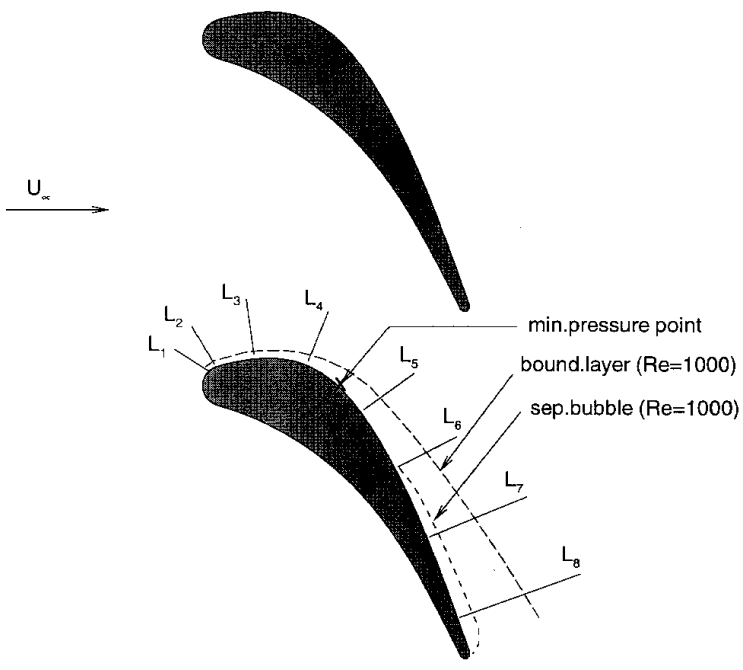

Fig. 4 Airfoil suction-side perpendicular stations

Fig. 5 Mean flow characteristics across boundary layer (a) L4; (b) L5; (c) L7

FIGURE 21. Mean flow characteristics across the boundary layer perpendicular stations. (a) $\mathrm{L}_{1}$; (b) $\mathrm{L}_{2}$; (c) $\mathrm{L}_{3} ;$ (d) $\mathrm{L}_{4} ;$ (e) $\mathrm{L}_{5}$; (f) $\mathrm{L}_{6} ;$ (g) $\mathrm{L}_{7} ;$ (h) $\mathrm{L}_{8}$. Basic flow at FIGURE 5. $\mathrm{Re}=1000$.

\section{LIST OF FIGURES}

\section{FIGURE 1.}

FIGURE 2.

FIGURE 3.

FIGURE 4.

Two-dimensional computational domain depicting relative dimensions and history points (P1, P2 and P3) locations. Lowsolidity $(\mathrm{h} / \mathrm{c}=0.914)$, experimental axial gas turbine first stator.

Typical spectral twodimensional mesh discretization including collocation points. 784 macroelements and 25 collocation points per macro-element.

Balance of Fluctuating Kinetic Energy in an elementary control volume. Includes the transport, production and dissipation terms.

Mean pressure contour plot for the basic flow at $R e=1000$.

Boundary layer thickness $\delta$ nondimensionalized by the blade chord $c$ as a function of $\mathrm{Re}$ and 
the airfoil suctionside arclength.

FIGURE 6.

FIGURE 7.

FIGURE 8.

FIGURE 9.

FIGURE 10.

FIGURE 11.

Streamtraces for the
basic flows at
$R e=231$ and
$\operatorname{Re}=1000$.

FIGURE 12.

FIGURE 13.

domain at a characteristic time for basic flow at $\mathrm{Re}=1000$.

Sp' vs. time at eight locations close to the suction-side wall $\left(d_{n} / c=0.0115\right) \quad$ along the streamwise direction. Basic flow at $R e=1000$.

Fourier power
spectrum of the $x-$
velocity at point $\mathrm{P} 1$.
Basic flow at
$\operatorname{Re}=1000$.

Wavelength modulation observed in Sp' vs. time plot along line close to the suction-side wall $\left(d_{n} / c=0.016\right)$ at three different times within one period of the fluctuation. Basic flow at $\mathrm{Re}=1000$.

Contour plot of Sp' within the airfoil-toairfoil section at four times within one period of the

FIGURE 15.

FIGURE 16. fluctuation. Basic flow at $R e=1000$.

Contour plot of Sp' within the airfoil-toairfoil section at four times within one period of the fluctuation. Basic flow at $R e=600$.

Comparison between the mean flow pressure coefficient $\mathrm{C}_{\mathrm{p}} \quad$ obtained numerically at $R e=1000$ and the one obtained

experimentally by Dring et al. (1987) at $\mathrm{Re}=5.9 \times 10^{5}$.

FIGURE 14. Stokes wave generated by the superposed

perturbation with $\omega_{\mathrm{d}}=26.4$ and $\varepsilon=0.02$. (a) carpet plot of $\mathrm{Sp}$; and (b) contour plots of $p$ at four times within the perturbation period.

Carpet plots of Sp' at the moment when inlet perturbation vanishes for subcritical perturbed flow. $R e=231$ and $\varepsilon=0.02$. (a) $\omega_{\mathrm{d}}=88.0$;

(b) $\omega_{\mathrm{d}}=26.4$.

Local measurement of the subcritical perturbed field at $\mathrm{Re}=231$. Subcritical 
flow perturbed with $\varepsilon=0.02$ and, $\omega_{\mathrm{d}}=88.0$ and $\omega_{\mathrm{d}}=26.4$. (a) Fourier power spectrum of the $x$ velocity at point $\mathrm{P} 1$; (b) Sp' vs. X along quasi-parallel lines to the suction-side wall.

FIGURE $17 . \quad$ Local measurement of the supercritical perturbed field at $\mathrm{Re}=1000, \quad \varepsilon=0.02$. Fourier power spectrum of $x$-velocity at point P1. (a) $\omega_{d}=88.0$; (b) $\omega_{d}=26.4$.

FIGURE 18. Carpet plot of Sp' at the moment when inlet perturbation vanishes for supercritical

perturbed flow, compared to basic flow at characteristic time for $R e=1000$, $\varepsilon=0.02$, and $\omega_{\mathrm{d}}=88.0$ and $\omega_{\mathrm{d}}=26.4$. throughout the entire computational

domain;

throughout airfoil suction-side

boundary layer.

FIGURE 19. Local receptivity measurement at point P1. Sp'amp vs. $\omega_{\mathrm{d}}, \varepsilon$ and Re. $(231 \leq \operatorname{Re}$ $\leq 1000 ; \quad 0.005 \leq \varepsilon$ $\leq 0.02 ; 8.8 \leq \omega \leq 1100)$.
FIGURE 20.

FIGURE 21.

FIGURE 21.

FIGURE 22.

FIGURE 23.

FIGURE 24.

FIGURE 25.
Airfoil suction-side perpendicular

stations where transverse parameter distributions are plotted.

Mean flow characteristics across the boundary layer perpendicular stations. (a) $\mathrm{L}_{1}$; (b) $\mathrm{L}_{2}$; (c) $\mathrm{L}_{3}$; (d) $\mathrm{L}_{4}$; (e) $\mathrm{L}_{5}$; (f) $\mathrm{L}_{6} ;$ (g) $\mathrm{L}_{7}$; (h) $\mathrm{L}_{8}$. Basic flow at $\mathrm{Re}=1000$.

Contour plot of T3 within the airfoil suction-side boundary layer. Comparison between: (a) directly-computed T3, and (b) T3 resulting from the balance of the FKE equation using $T 1=0$. Basic flow at $\mathrm{Re}=1000$.

FKE budget across boundary layer perpendicular stations. Basic flow at $\mathrm{Re}=1000$.

Reynolds shear

stress

distribution across boundary layer perpendicular

stations. Basic flow at $\mathrm{Re}=1000$.

Contour plots of FKE terms within strip 
enclosing the region where T-S waves generate. Basic flow at $\mathrm{Re}=1000$. (a) T2; (b) T3; (c) T4.

FIGURE 26.

FIGURE 27.

FIGURE 28.

FIGURE 29.
Changes in the mean flow, measured across boundary layer perpendicular stations. Subcritical perturbed flow at $\mathrm{Re}=231$ with $\varepsilon=0.02$, and $\omega_{\mathrm{d}}=88.0$ and $\omega_{\mathrm{d}}=26.4$. (a) $\mathrm{L}_{2}$; (b) $\mathrm{L}_{4}$; (c) $\mathrm{L}_{5}$; (d) $\mathrm{L}_{7}$; (e) $\mathrm{L}_{8}$.

Changes in the FKE terms, measured across boundary layer perpendicular stations. Subcritical perturbed flow at $\mathrm{Re}=231$ with $\varepsilon=0.02$, and $\omega_{\mathrm{d}}=88.0$ and $\omega_{\mathrm{d}}=26.4$. (a) $\mathrm{L}_{2}$; (b) $\mathrm{L}_{4}$; (c) $\mathrm{L}_{5} ;$ (d) $\mathrm{L}_{7} ;$ (e) $\mathrm{L}_{8}$.

FIGURE 32.

Changes in the mean flow, measured across boundary layer perpendicular stations. Supercritical perturbed flow at $\mathrm{Re}=1000$ with $\varepsilon=0.02$, and $\omega_{\mathrm{d}}=88.0$ and $\omega_{\mathrm{d}}=26.4$. (a) $\mathrm{L}_{2}$; (b) $\mathrm{L}_{4}$; (c) $\mathrm{L}_{5} ;$ (d) $\mathrm{L}_{7}$; (e) $\mathrm{L}_{8}$.

Changes in the FKE terms, measured across boundary layer perpendicular stations. Supercritical perturbed flow at

FIGURE 31.

FIGURE 33.
$\mathrm{Re}=1000$ with $\varepsilon=0.02$, and $\omega_{\mathrm{d}}=88.0$ and $\omega_{\mathrm{d}}=26.4$. (a) $\mathrm{L}_{2}$; (b) $\mathrm{L}_{4}$; (c) $L_{5} ;$ (d) $L_{7} ;(e) L_{8}$.

FIGURE 30.

Three-dimensional computational

domain. (a) mesh with 630 macroelements; (b) open mesh depicting macro-elements and $5 \times 5 \times 5$ nodes per macro-element.

Flow field at midspan section. Comparison between threedimensional and twodimensional

simulations. Basic flow at $R e=400$. (a) streamtraces based on $x$ - and $y$-velocity components; mean pressure field.

Carpet plot of Sp' at midspan section at characteristic time. Three-dimensional simulation of basic flow at $R e=400$.

Carpet plots of Sp' at midspan section. Three-dimensional simulations at $\mathrm{Re}=400$. Comparison between basic flow at characteristic time and perturbed flow with $\varepsilon=0.02$ and $\omega_{\mathrm{d}}=26.4$ when the 
inlet perturbation

vanishes. 